\title{
Homotopy Perturbation Method for Solving Moving Boundary and Isoperimetric Problems
}

\author{
Sara Ghaderi \\ Kavian University, Mashhad, Iran \\ Email: s_gh333@yahoo.com
}

Received January 3, 2012; revised March 19, 2012; accepted March 26, 2012

\begin{abstract}
In this paper, homotopy perturbation method is applied to solve moving boundary and isoperimetric problems. This method does not depend upon a small parameter in the equation, homotopy is constructed with an imbedding parameter $p$, which is considered as a "small parameter". Finally, we use combined homotopy perturbation method and Green's function method for solving second order problems. Some examples are given to illustrate the effectiveness of methods. The results show that these methods provides a powerful mathematical tools for solving problems.
\end{abstract}

Keywords: Homotopy Perturbation Method; Moving Boundaries; Isoperimetric Problems

\section{Introduction}

In the modeling of a large class of problems which comes up in science, engineering and economics, it is necessary to minimize amounts of a certain functional. Because of the key role of this subject, it has been considerable attention has been devoted to these kinds of problems. Such problems are called variational problems (see [1,2]).

Consider the simplest form of a variational problem as:

$$
v[y(x)]=\int_{x_{0}}^{x_{1}} F\left(x, y(x), y^{\prime}(x)\right) \mathrm{d} x,
$$

where $v$ is the functional that its extremum must be achieved. There are two kinds of boundary conditions that functional $v$ can be considered by. In the case of fixed boundary problems, the admissible function $y(x)$ must satisfy the boundary conditions

$$
y\left(x_{0}\right)=y_{0}, y\left(x_{1}\right)=y_{1}
$$

In moving boundary problems at least one of the boundary points of the admissible function is movable along a boundary curve. As a matter of fact, many applications of the calculus of variations lead to problems in which not only boundary conditions, but also conditions of quite a different type, known as constraints are imposed on the admissible function. The necessary condition for the admissible solutions at this problems is to satisfy the Euler-Lagrange equation which is mainly consider as nonlinear.

In this work we consider Homotopy perturbation method, which is an effective and applicable mathematical tool for linear and nonlinear equations. It yields a rapid convergence of the solution, and doesn't have previous perturbation method limits (see [3-12]).

Author of [13] solved variational problems with moving boundaries with Adomian decomposition method.

In [14] Homotopy perturbation method applied to solve variational problems with fixed boundaries. In this paper solution of variational problems with moving boundaries problems can be obtained by Homotopy perturbation method first. Then we obtain solution of them by using combined homotopy perturbation method and Green's function method. This algorithm is offered for the solution of second-order boundary value problems with two-point boundary conditions. To transform the ordinary differential equation into an equivalent integral one, which has already satisfied the boundary conditions, we apply the Green's function method first. Then, the homotopy perturbation method is used to the resulting equation to construct the numerical solution for such problems. To illustrate a clear overview of the procedure several illustrative examples are involved.

\section{Statement of the Problem}

\subsection{Moving Boundary Problems}

The essential condition for the solution of problem (1) has been fulfilled the Euler-Lagrange equation

$$
\frac{\partial F}{\partial y}-\frac{\mathrm{d}}{\mathrm{d} x} \frac{\partial F}{\partial y^{\prime}}=0
$$

The general form of the variational problem (1) is 


$$
v\left[y_{1}, y_{2}, \cdots, y_{n}\right]=\int_{x_{0}}^{x_{1}} F\left(x, y_{1}, y_{2}, \cdots, y_{n}, y_{1}^{\prime}, \cdots, y_{n}^{\prime}\right) \mathrm{d} x,
$$

Here the necessary condition for the extremum of the functional (4) is satisfying the system of second-order differential equations below

$$
\frac{\partial F}{\partial y_{i}}-\frac{\mathrm{d}}{\mathrm{d} x} \frac{\partial F}{\partial y_{i}^{\prime}}=0, i=1,2, \cdots, n
$$

In the fixed boundary problems, Euler-Lagrange equation must be considered by the boundary conditions, but for the problems with variable boundaries, Euler-Lagrange equation has to satisfy natural boundary conditions or transversality conditions that has been discussed in the following theorems.

Type 1: Firstly, we consider problems for which at least one of the boundary points move freely along a line parallel to the $y$-axis, actually at this point $y(x)$ is not specified. In this case all admissible functions have the same domain of definition $\left[x_{0}, x_{1}\right]$ and satisfy the EulerLagrange equation in this interval. Furthermore such functions must satisfy conditions called natural boundary conditions prescribed in the following theorem.

Theorem 2.1. Suppose the function $y=y(x)$ in $C^{1}\left[x_{0}, x_{1}\right]$, yields a relative minimum of the functional (1) for which $y\left(x_{0}\right)=y_{0}$ is given, $y\left(x_{1}\right)=y_{1}$ is arbitrary (free right endpoint) or $y\left(x_{0}\right), y\left(x_{1}\right)$ are arbitrary (free endpoints).

Then $y_{0}(x)$ satisfies, respectively, the following natural boundary conditions:

$$
\frac{\partial F}{\partial y^{\prime}}\left(x_{1}, y_{0}\left(x_{1}\right), y_{0}^{\prime}\left(x_{1}\right)\right)=0 \text {, }
$$

Or

$$
\begin{aligned}
& \frac{\partial F}{\partial y^{\prime}}\left(x_{0}, y_{0}\left(x_{0}\right), y_{0}^{\prime}\left(x_{0}\right)\right) \\
& =\frac{\partial F}{\partial y^{\prime}}\left(x_{1}, y_{0}\left(x_{1}\right), y_{0}^{\prime}\left(x_{1}\right)\right)=0
\end{aligned}
$$

Type 2: Secondly, we ought to turn to the beginning and end points (or only one of them) that move freely on given curves $y=\varphi(x), y=\psi(x)$. In this case, we look for a function $y(x)$, which emanates at some $x=x_{0}$ from the curve $y=\varphi(x)$ and terminates for some $x=x_{1}$ on the curve $y=\psi(x)$ and minimizes the functional (1). In this problem the points $x_{0}, x_{1}$ are unknown, they must satisfy the necessary conditions called transversality conditions, prescribed in the following theorem.

Theorem 2.2. If the function $y=y_{0}(x) \in C^{1}\left[x_{0}, x_{1}\right]$, which emanates at some $x=x_{0}$ from the curve $y=\varphi(x) \in C^{1}(-\infty,+\infty)$ and terminates for some $x=x_{1}$ on the curve $y=\psi(x) \in C^{1}(-\infty,+\infty)$, yields a relative minimum for functional (1), where $F \in C^{1}(R), R$ being a domain in the $\left(x, y, y^{\prime}\right)$ space that contains all lineal elements of $y=y_{0}(x)$, then it is necessary that $y=y_{0}(x)$ must satisfy the Euler-Lagrange equation in the interval $\left[x_{0}, x_{1}\right]$ and that at the point of departure and the point of arrival, the transversality conditions:

$$
\begin{aligned}
& \frac{\partial F}{\partial y^{\prime}}\left(x_{0}, y_{0}\left(x_{0}\right), y_{0}^{\prime}\left(x_{0}\right)\right)\left(\Phi^{\prime}\left(x_{0}\right)-y_{0}^{\prime}\left(x_{0}\right)\right) \\
& +F\left(x_{0}, y_{0}\left(x_{0}\right), y_{0}^{\prime}\left(x_{0}\right)\right)=0, \\
& \frac{\partial F}{\partial y^{\prime}}\left(x_{1}, y_{0}\left(x_{1}\right), y_{0}^{\prime}\left(x_{1}\right)\right)\left(\psi^{\prime}\left(x_{1}\right)-y_{0}^{\prime}\left(x_{1}\right)\right) \\
& +F\left(x_{1}, y_{0}\left(x_{1}\right), y_{0}^{\prime}\left(x_{1}\right)\right)=0,
\end{aligned}
$$

are satisfied. In such a state that one of the points is fixed, then the transversality condition has to hold at the other point. One can consider transversality conditions for the problems with more than one unknown functions. For example, in the two dimensional case we seek a vector function $y(x)=\left(y_{1}(x), y_{2}(x)\right)$ as minimizes

$$
v\left[y_{1}, y_{2}\right]=\int_{x_{0}}^{x_{1}} F\left(x, y_{1}, y_{2}, y_{1}^{\prime}, y_{2}^{\prime}\right) \mathrm{d} x,
$$

in which $y_{1}\left(x_{0}\right)=y_{1, x_{0}}, y_{2}\left(x_{0}\right)=y_{2, x_{0}}$ and the endpoint lies on a two-dimensional surface that is given by $x=u\left(y_{1}, y_{2}\right)$. Here the transversality conditions at $x=x_{1}$ are:

$$
\begin{aligned}
& \left(\frac{\partial u}{\partial y_{1}} F+\left(1-\frac{\partial u}{\partial y_{1}} y_{1}^{0^{\prime}}-\frac{\partial u}{\partial y_{2}} y_{2}^{0^{\prime}}\right) \frac{\partial u}{\partial y_{1}^{\prime}} F\right)\left(x_{1}\right)=0, \\
& \left(\frac{\partial u}{\partial y_{2}} F+\left(1-\frac{\partial u}{\partial y_{1}} y_{1}^{0^{\prime}}-\frac{\partial u}{\partial y_{2}} y_{2}^{0^{\prime}}\right) \frac{\partial u}{\partial y_{2}^{\prime}} F\right)\left(x_{1}\right)=0,
\end{aligned}
$$

In which $\left(y_{1}^{0}(x), y_{2}^{0}(x)\right)$ is an admissible vector function.

For more information on transversality conditions, specially for the proofs of Theorems 2.1 and 2.2 and conditions (11), (12) (see [15]).

\subsection{Isoperimetric Problems}

Assume that two functions $G\left(x, y, y^{\prime}\right)$ and $F\left(x, y, y^{\prime}\right)$ are given. From among all curves $y=y(x) \in C^{1}\left[x_{0}, x_{1}\right]$ along which the functional

$$
K[y]=\int_{x_{0}}^{x_{1}} G\left(x, y, y^{\prime}\right) \mathrm{d} x
$$

assumes a given value $l$, determine the one for which the functional

$$
J[y]=\int_{x_{0}}^{x_{1}} F\left(x, y, y^{\prime}\right) \mathrm{d} x
$$

assumes an extremal value. We assume that $F$ and $G$ have continuous first and second partial derivatives for $x_{0} \leq x \leq x_{1}$ and for arbitrary values of the variables $y$ and $y^{\prime}$.

Euler's theorem: If a curve $y=y(x)$ extremizes the 
functional $J[y]=\int_{x_{0}}^{x_{1}} F\left(x, y, y^{\prime}\right) \mathrm{d} x$ subject to the conditions

$$
K[y]=\int_{x_{0}}^{x_{1}} G\left(x, y, y^{\prime}\right) \mathrm{d} x=l, y\left(x_{0}\right)=y_{0}, y\left(x_{1}\right)=y_{1}
$$

and if $y=y(x)$ is not an extremal of the functional $K$, then there exists a constant $\lambda$, such that the curve $y=y(x)$ is an extremal of the functional

$$
L=\int_{x_{0}}^{x_{1}}\left[F\left(x, y, y^{\prime}\right)+\lambda G\left(x, y, y^{\prime}\right)\right] \mathrm{d} x
$$

The vital condition for the solution of this problem is to satisfy the Euler-Lagrange equation

$$
\frac{\partial H}{\partial y}-\frac{\mathrm{d}}{\mathrm{d} x} \frac{\partial H}{\partial y^{\prime}}=0
$$

with given boundary conditions in which $H=F+\lambda G$, see [15] for further information.

\section{Homotopy Perturbation Method}

We consider the following nonlinear differential equation

$$
A(y)-f(r)=0, r \in \Omega
$$

with natural boundary conditions or transversality conditions

$$
B\left(y, \frac{\partial y}{\partial n}\right)=0, r \in \Gamma
$$

where $A$ is a general differential operator, $B$ is a boundary operator, $f(r)$ is a known analytic function and $\Gamma$ is the boundary of the domain $\Omega$.

The operator $A$ can, generally, be divided into two parts $L$ and $N$, where $L$ is Linear, while $N$ is nonlinear, so that we can write

$$
L(y)+N(y)-f(r)=0 .
$$

By Homotopy perturbation technique [3] and [4], we create a homotopy $v(r, p): \Omega \times[0,1] \rightarrow R$ which satisfies

$$
\begin{aligned}
& H(v, p)=(1-p)\left[L(v)-L\left(y_{0}\right)\right] \\
& +p[A(v)-f(r)]=0, p[0,1], r \in \Omega .
\end{aligned}
$$

Or

$$
\begin{aligned}
& H(v, p)=L(v)-L\left(y_{0}\right)+p L\left(y_{0}\right) \\
& +p[N(v)-f(r)]=0 .
\end{aligned}
$$

where $p \in[0,1]$ is an embeding parameter, and $y_{0}$ is an initial approximation of Equation (13). Obviously from Equation (16):

$$
\begin{aligned}
& H(v, 0)=L(v)-L\left(y_{0}\right)=0, \\
& H(v, 1)=A(v)-f(r)=0,
\end{aligned}
$$

$v(r, p)$ have to change from $y_{0}(r)$ to $y(r)$ due to the changing process of $p$ from zero to unity. In topology, this is named deformation, and $L(v)-L\left(y_{0}\right), A(v)-f(r)$ are called homotopic.

In this method, using the homotopy parameter $p$, we have the following power series in $p$

$$
v=v_{0}+p v_{1}+p^{2} v_{2}+\cdots
$$

Setting $p=1$ results in the approximate solution of Equation (13)

$$
y=\lim _{p-1} v=v_{0}+v_{1}+v_{2}+\cdots
$$

\section{Numerical Examples}

Example 3.1.1. Consider the following problems:

$$
J[y]=\int_{0}^{T} a\left(b y(t)-y^{\prime}(t)-c^{*}\right)^{2} \mathrm{~d} t
$$

In which $a, b>0, c^{*}>0$ and $y(t)$ is the amount of a capital at time $t$. Here, the capital stock $y(0)$ at the initial time $t=0$, of the planning period is pretended to be known: $y(0)=y_{0}$, on the other hand, the planner will not want to prescribe how large the capital will be at time $t=T$. Hence, there exists a variational problem with free right endpoint. Here we let $a=b=c^{*}=1$, $T=1$, and $y_{0}=2$ which has the analytical solution $y(t)=1+e^{t}$.

The Euler-Lagrange equation for this problem is:

$$
y^{\prime}(t)-y(t)+1=0 .
$$

We know the natural boundary condition at $t=1$ is

$$
\frac{\partial f}{\partial y^{\prime}}\left(1, y(1), y^{\prime}(1)\right)=-2\left(y(t)-y^{\prime}(t)-1\right)_{t=1}=0 .
$$

Therefore, we have the following boundary conditions for (3.1.2):

$$
y(0)=2, y(1)-y^{\prime}(1)-1=0 .
$$

We can readily construct a homotopy which satisfies

$$
\begin{aligned}
& v^{\prime \prime}(t)-v(t)-y_{0}^{\prime \prime}(t)+y_{0}(t)+p y_{0}^{\prime \prime}(t) \\
& -p y_{0}(t)+p=0
\end{aligned}
$$

With initial approximation $y_{0}(t)=a e^{t}$, Suppose that the solution of Equation (25) has the (20).

Substituting (20) into (25), and equating the terms with the identical powers of $p$ :

$$
\begin{aligned}
& v_{0}^{\prime \prime}(t)-v_{0}(t)-y^{\prime \prime}(t)-y_{0}(t)=0, \\
& v_{1}^{\prime \prime}(t)-v_{1}(t)+y^{\prime \prime}(t)-y_{0}(t)+1=0, \\
& v_{2}^{\prime \prime}(t)-v_{2}(t)=0 .
\end{aligned}
$$

assuming $v_{0}(t)=y_{0}(t)=a e^{t}$, we have $v_{1}(t)=b+c e^{t}$, and $v_{2}(t)=d e^{t}$. So, for Equation (22) we have an approximate solution: 


$$
y=v_{0}+v_{1}+v_{2}=b++(a+c+d) e^{t} .
$$

Imposing (24) at (26), we have: $y(t)=1+e^{t}$.

Now we solve this problem with Adomian decomposition method. Using the operator form of (23), we have:

$$
\begin{aligned}
& L y=y-1, \\
& y(x)=2+A x-L^{-1}(1)+L^{-1}(y(x)) .
\end{aligned}
$$

Applying the Adomian decomposition:

$$
\sum_{n=0}^{\infty} y_{n}(x)=2+A x-\frac{x^{2}}{2}+L^{-1}\left(\sum_{n=0}^{\infty} y_{n}(x)\right) .
$$

So, we use the reccursive relations

$$
\begin{aligned}
& y_{0}(x)=2+A x-\frac{x^{2}}{2}, \\
& y_{n+1}(x)=L^{-1}\left(y_{n}(x)\right) .
\end{aligned}
$$

Now we use $g_{n}(x)$ as the approximation of $y(x)$, for example, for $n=3$ we have:

$$
\begin{aligned}
g_{3}(x)= & 2+A x+\frac{x^{2}}{2}+\frac{A x^{3}}{6}+\frac{x^{4}}{24} \\
& +\frac{A x^{5}}{120}+\frac{x^{6}}{720}+\frac{A x^{7}}{5040}-\frac{x^{8}}{40320}
\end{aligned}
$$

We solve the equation $g_{3}(1)-g_{3}^{\prime}(1)-1=0$, for the determination of $A$. Table 1 shows the error of $g_{3}$.

Solutions of two methods show that the homotopy perturbation method is better than adomian decomposition method.

Example 3.1.2. We want to find the minimum of the integral $J[y(x)]=\int_{0}^{1} y^{\prime 2}(x) \mathrm{d} x$ subject to the conditions

$$
\int_{0}^{1} y(x) \mathrm{d} x=3, y(0)=1, y(1)=6 .
$$

From the auxiliary function [15]:

$$
\int_{0}^{1}\left(y^{\prime 2}(x)+\lambda y(x)\right) \mathrm{d} x
$$

\begin{tabular}{|c|c|c|c|c|c|}
\hline$x 0$ & 0.2 & 0.4 & 0.6 & 0.8 & 1 \\
\hline
\end{tabular}

A homotopy can be constructed as follows

$$
v^{\prime \prime}(t)-y_{0}^{\prime \prime}(t)+p y_{0}^{\prime \prime}(t)-p \frac{\lambda}{2}=0 .
$$

Our initial approximation is: $y_{0}(t)=a t+b$. Supposing the approximate solution of Equation (29) has the form of (20), by the same manipulation like above example, we have

$$
v_{0}^{\prime \prime}(t)-y_{0}^{\prime \prime}(t)=0,
$$

Table 1. Shows the error of $g_{3}$.

$$
\begin{aligned}
& v_{1}^{\prime \prime}(t)+y_{0}^{\prime \prime}(t)-\frac{\lambda}{2}=0, \\
& v_{2}^{\prime \prime}(t)=0 .
\end{aligned}
$$

We suppose that $v_{0}(x)=y_{0}(t)=a x+b$. So $v_{1}(t)=\frac{\lambda}{4} t^{2}+c t+d, v_{2}(t)=e t+f, \cdots$

Therefore we find the following equation

$$
v(x)=\frac{\lambda}{4} t^{2}+(a+c+e) t+(b+d+f)
$$

Imposing $y(0)=1, y(1)=6, \int_{0}^{1} y(x) \mathrm{d} x=3$ on (30) we have:

$$
\lambda=12, y(x)=3 x^{2}+2 x+1
$$

\section{Combined Homotopy Perturbation Method and Green's Function Method for Solving Second-Order Moving Boundary Problems}

Assume that [16]

$$
y^{\prime \prime}(x)+\lambda^{2} y(x)+f\left[y(x), y^{\prime}(x)\right]=F(x), 0<x<l
$$

with boundary conditions that obtained from transversality conditions. Where $\lambda$ is a real constant number, $f\left(y, y^{\prime}\right)$ is a nonlinear function, and $F(x)$ is a nonhomogeneous term.

We can write Equation (32) to an equivalent integral equation;

$$
y(x)=-\int_{0}^{1}\left\{F(\xi)-f\left[y(\xi), y^{\prime}(\xi)\right]\right\} G(x, \xi) \mathrm{d} \xi,
$$

By adding a nonhomogeneous term, in moving boundary problems, the above equation is modified because boundary conditions are nonhomogeneous. Here, the function $G(x, \xi)$ the function $G(x, \xi)$ that called Green's function, is adjusted as below:

$$
\begin{aligned}
& G(x, \xi) \\
& =\frac{1}{\lambda \sin (\lambda l)} \begin{cases}\sin (\lambda \xi) \sin (\lambda(l-x)), & 0 \leq \xi \leq x \\
\sin (\lambda x) \sin (\lambda(l-\xi)), & x \leq \xi \leq l .\end{cases}
\end{aligned}
$$

In Equation (33), if $\lambda=0$, then Green's function amended as below:

$$
G(x, \xi)=\frac{1}{l} \begin{cases}\xi(l-x), & 0 \leq \xi \leq x \\ x(l-\xi), & x \leq \xi \leq l .\end{cases}
$$

We construct the homotopy form of Equation (33), which satisfies:

$$
\begin{aligned}
& H(v, p)=v(x)+\int_{0}^{1} F(\xi) G(x, \xi) \mathrm{d} \xi \\
& -p \int_{0}^{1} f\left[v(\xi), v^{\prime}(\xi)\right] G(x, \xi) \mathrm{d} \xi=0, p \in[0,1] .
\end{aligned}
$$

The solution of the Equation (32) is assumed to be the 
power series of $p$ as expressed in Equation (20) and substituting Equation (20) into (36) and equating the term with identical powers of $p$, The following equations can

$$
p^{i}: v_{i}(x)=-\int_{0}^{1} f\left[v_{i-1}(\xi), v_{i-2}(\xi), \cdots, v_{0}(\xi), v_{i-1}^{\prime}\right.
$$

We obtain the approximate solution from Equation (20) by solving these integral equation. If we construct the homotopy as:

$$
\begin{aligned}
& H(v, p)=v(x)+\int_{0}^{1} F(\xi) G(x, \xi) \mathrm{d} \xi \\
& -\int_{0}^{1}\left\{(1-p) f\left[y_{\text {exa }}(\xi), y_{\text {exa }}^{\prime}(\xi)\right]+p f\left[v(\xi), v^{\prime}(\xi)\right]\right\} \\
& \cdot G(x, \xi) \mathrm{d} \xi=0, p \in[0,1] .
\end{aligned}
$$

where $y_{\text {exa }}(x)$ is the exact solution of (32), then we have:

$$
\begin{aligned}
v_{0}(x) & =-\int_{0}^{1} F(\xi) G(x, \xi) \mathrm{d} \xi \\
& +\int_{0}^{1} f\left[y_{\text {exa }}(\xi), y_{\text {exa }}^{\prime}(\xi)\right] G(x, \xi) \mathrm{d} \xi
\end{aligned}
$$

Equation (38) will give the exact solution.

\section{Numerical Examples}

Example 4.1.1. Considering Ramsey growth model, we have:

$$
\begin{aligned}
y_{i}(x)= & a x+b+\int_{0}^{1} G(x, \xi) \mathrm{d} \xi \\
& -\int_{0}^{1} y_{i-1}(\xi) G(x, \xi) \mathrm{d} \xi=0
\end{aligned}
$$

Also we have:

$$
\begin{aligned}
& p^{0}: v_{0}(x)=a x+b+\int_{0}^{1} G(x, \xi) \mathrm{d} \xi=a x+b-\frac{1}{2} x^{2}+\frac{1}{2} x, \\
& p^{1}: v_{1}(x)=-\int_{0}^{1} v_{0} G(x, \xi) \mathrm{d} \xi=-\frac{1}{2} b x \\
& +\left(\frac{1}{2} b-\frac{1}{2} a-\frac{1}{4}\right) x^{2}+\left(\frac{1}{2} a+\frac{1}{2}\right) x^{3}-\frac{1}{4} x^{4}, \\
& p^{2}: v_{2}(x)=-\int_{0}^{1} v_{1} G(x, \xi) \mathrm{d} \xi \\
& =\frac{1}{4} b x^{2}+\left(-\frac{1}{2} b+\frac{1}{4} a+\frac{1}{8}\right) x^{3} \\
& +\left(\frac{1}{4} b-\frac{1}{2} a-\frac{3}{8}\right) x^{4}+\left(\frac{1}{4} a+\frac{3}{8}\right) x^{5}-\frac{1}{8} x^{6}, \\
& p^{3}: v_{3}(x)=-\int_{0}^{1} v_{2} G(x, \xi) \mathrm{d} \xi=-\frac{1}{8} b x^{3} \\
& +\left(\frac{3}{8} b-\frac{1}{4} a-\frac{1}{16}\right) x^{4}+\left(-\frac{3}{8} b+\frac{3}{8} a+\frac{1}{4}\right) x^{5} \\
& +\left(\frac{1}{8} b-\frac{3}{8} a-\frac{3}{8}\right) x^{6}+\left(\frac{1}{8} a+\frac{1}{4}\right) x^{7}-\frac{1}{16} x^{8},
\end{aligned}
$$

be obtained:

$$
p^{0}: v_{0}(x)=-\int_{0}^{1} F(\xi) G(x, \xi) \mathrm{d} \xi,
$$

$\left.(\xi), v_{i-2}^{\prime}(\xi), \cdots, v_{0}^{\prime}(\xi) G(x, \xi)\right] \mathrm{d} \xi,(i=1,2,3, \cdots)$

$$
v_{i}(x)=-\int_{0}^{1} v_{i-1} G(x, \xi) \mathrm{d} \xi,(i=4,5,6, \cdots) .
$$

Assume that $r_{1}(x)=v_{0}(x)+v_{1}(x)$ and by imposing boundary condition on that, we have:

$$
a=1, b=2 \text {, }
$$

So:

$$
r_{1}(x)=2+\frac{1}{2} x-\frac{1}{4} x^{2}+x^{3}-\frac{1}{4} x^{4} .
$$

And assuming $r_{2}(x)=v_{0}(x)+v_{1}(x)+v_{2}(x)$ and imposing boundary condition on that, we have:

$$
a=1, b=2 \text {, }
$$

So:

$$
r_{2}(x)=2+\frac{1}{2} x+\frac{1}{4} x^{2}+\frac{3}{8} x^{3}-\frac{5}{8} x^{4}+\frac{5}{8} x^{5}-\frac{1}{8} x^{6} .
$$

Also we can write:

$$
\begin{aligned}
r_{3}(x)= & 2+\frac{1}{2} x+\frac{1}{4} x^{2}+\frac{1}{8} x^{3}-\frac{1}{16} x^{4}+\frac{1}{2} x^{5} \\
& -\frac{5}{8} x^{6}+\frac{3}{8} x^{7}-\frac{1}{16} x^{8} .
\end{aligned}
$$

But from (37) we have:

$$
\begin{aligned}
v(x) & =x+2+\int_{0}^{1} G(x, \xi) \mathrm{d} \xi \\
& -\int_{0}^{1}\left\{\left(1+e^{\xi}\right)+p v(\xi)\right\} G(x, \xi) \mathrm{d} \xi .
\end{aligned}
$$

So:

$$
\begin{aligned}
& v_{0}(x)=x+2+\int_{0}^{1} G(x, \xi) \mathrm{d} \xi \\
& -\int_{0}^{1}\left(1+e^{\xi}\right) G(x, \xi) \mathrm{d} \xi \\
& =1+2 x+e^{x}-x e .
\end{aligned}
$$

That is approach to exact solution. And also we obtain:

$$
v_{i}(x)=0, i=1,2, \cdots \text {. }
$$

The plot of $v_{0}(x), r_{1}(x), r_{2}(x), r_{3}(x)$ has been shown

\section{in Figure 1.}

Example 4.1.2. We want to solve Example 3.1.2 with this method. The Euler-Lagrange equation is:

$$
y^{\prime \prime}-\frac{\lambda}{2}=0 \text {. }
$$

Supposing $v(x)=a x+b$ we have: 


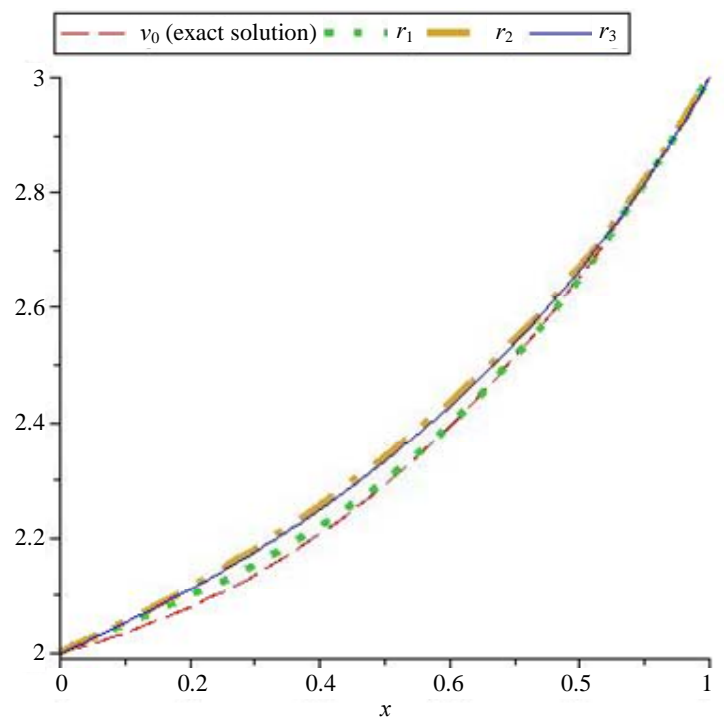

Figure 1. The plot of $v_{0}(x), r_{1}(x), r_{2}(x), r_{3}(x)$ of Example 4.1.1.

$$
\begin{aligned}
G(x, \xi) & =\frac{1}{l} \begin{cases}x(l-\xi) & x \leq \xi \leq l \\
\xi(l-x) & 0 \leq \xi \leq x\end{cases} \\
& = \begin{cases}x(1-\xi) & x \leq \xi \leq 1 \\
\xi(1-x) & 0 \leq \xi \leq x\end{cases}
\end{aligned}
$$

and

$$
\begin{aligned}
y(x) & =a x+b-\int_{0}^{1} F(\xi) G(x, \xi) \mathrm{d} \xi \\
& +p \int_{0}^{1} f\left[y(\xi), y^{\prime}(\xi)\right] G(x, \xi) \mathrm{d} \xi \\
& =a x+b-\int_{0}^{1} \frac{\lambda}{2} G(x, \xi) \mathrm{d} \xi,
\end{aligned}
$$

So

$$
\begin{aligned}
& p^{0}: v_{0}(x)=a x+b-\int_{0}^{1} \frac{\lambda}{2} G(x, \xi) \mathrm{d} \xi \\
& =\frac{1}{4} \lambda x^{2}+\left(a-\frac{\lambda}{4}\right) x+b,
\end{aligned}
$$

$$
p^{i}: v_{i}(x)=-\int_{0}^{1} f\left[v_{i-1}(\xi), v_{i-2}(\xi), \cdots, v_{0}(\xi), v_{i-1}^{\prime}(\xi), v_{i-2}^{\prime}(\xi), \cdots, v_{0}^{\prime}(\xi) G(x, \xi)\right] \mathrm{d} \xi=0,(i=1,2,3, \cdots)
$$

by imposing boundary condition on that, we have:

$$
a=5, b=1, \lambda=12, y(x)=3 x^{2}+2 x+1 \text {. }
$$

\section{Conclusion}

In this paper, we solve the moving boundary and isoperimetric problems by using Homotopy perturbation method. Embedding parameter $p \in[0,1]$ can be taken into account as a perturbation parameter. By the application of Green's function, the problem concerned is transformed into an equivalent integral equation, which is solved using the homotopy perturbation method. Numerical examples show that these proposed methods were valid and effective for solving problems.

\section{REFERENCES}

[1] U. Brechtken-Manderschied, "Introduction to the Calculus of Variations,” Chapman Hall, London, 1991.

[2] H. Sagan, "Introduction to the Calculus of Variations," Dover Publications, New York, 1992.

[3] J.-H. He, "A Coupling Method for a Homotopy Technique and a Perturbation Technique for Nonlinear Problems," International Journal of Non-Linear Mechanics, Vol. 35, 2000, pp. 37-43.

[4] U. Ascher, M. Robert, M. M. Mattheij and R. D. Russell, "Numerical Solution of Boundary Value Problems for Ordinary Differential Equations,” SIAM, Philadelphia, 1995.

[5] G. Engstrom and U. Brechtken-Manderschied, "Introdu- ction to the Calculus of Variations," Chapman and Hall/CRC, London, 1991.

[6] D. D. Ganji, H. Tari and M. Bakhshi, "Variational Iteration Method and Homotopy Perturbation Method for Nonlinear Evalution Equations," Computers \& Mathematics with Applications, Vol. 54, No. 7-8, 2007, pp. 1018-1024.

[7] J. H. He, "Perturbation Method: A New Nonlinear Analytical Technique," Applied Mathematics and Computation, Vol. 135, No. 1, 2003, pp. 73-79. doi:10.1016/S0096-3003(01)00312-5

[8] M. Inokuti, H. Sekine and T. Mura, "General Use of the Lagrange Multiplier in Non-Linear Mathematical Physics,” In: S. Nemat-Nassed, Ed., Variational Methods in the Mechanics of Solids, Pergamon Press, New York, 1978, pp. 156-162.

[9] S. J. Liao, “An Approximate Solution Technique Not Depending on Small Parameters: A Special Example,” International Journal of Non-Linear Mechanics, Vol. 30, No. 3, 1995, pp. 371-380. doi:10.1016/0020-7462(94)00054-E

[10] S. J. Liao, "Boundary Element Method for General Nonlinear Differential Operators," Engineering Analysis with Boundary Element, Vol. 20, No. 2, 1997, pp. 91-99. doi:10.1016/S0955-7997(97)00043-X

[11] J. H. He, “Asymptotology by Homotopy Perturbation Method," Applied Mathematics and Computation, Vol. 156, No. 3, 2004, pp. 591-596. doi:10.1016/j.amc.2003.08.011

[12] J. H. He, “Homotopy Perturbation Technique,” Applied Mechanics and Engineering, Vol. 178, 1997, pp. 257262. 
[13] R. Memarbashi, "Variational Problems with Moving Boundaries Using Decomposition Method," Mathematical Problems in Engineering, Vol. 2007, 2007, Article ID 10120. doi:10.1155/2007/10120

[14] J. H. He, "Homotopy Perturbation Method for Solving Boundary Value Problems," Physics Letters A, Vol. 350, No. 1-2, 2006, pp. 87-88.

[15] M. L. Krasnov, G. I. Makarenko and A. L. Kiselev,
"Problems and Exercises in the Calculus of Variations," George Yankovsky, Moscow, 1975.

[16] Y.-G. Wang, H.-F. Song and D. Li, "Solving Two-Point Boundary Value Problems Using Combined Homotopy Perturbation Method and Green's Function Method," $A p$ plied Mathematics and Computation, Vol. 212, No. 2, 2009, pp. 366-376. 